\title{
Oxidative nucleophilic substitution of hydrogen in nitroarenes with an oxazoline-stabilized carbanion
}

\author{
Saverio Florio, ${ }^{* \mathrm{a}}$ Mieczyslaw Makosza, ${ }^{\mathrm{b}}$ Patrizia Lorusso, ${ }^{\mathrm{c}}$ and Luigino Troisi ${ }^{\mathrm{c}}$ \\ ${ }^{a}$ Dipartimento Farmaco-Chimico, University of Bari, Via E. Orabona 4, 70126 Bari, Istituto di \\ Chimica dei Composti Organometallici “ICCOM”, Sezione di Bari, Italy; ${ }^{b}$ Institute of Organic \\ Chemistry, Polish Academy of Sciences ul. Kasprzaka 44, 01-224 Warszawa 42, POB 58, \\ Poland; ${ }^{c}$ Dipartimento di Scienze e Tecnologie Biologiche ed Ambientali, University of \\ Lecce,Via Prov. le Lecce-Monteroni, 73100 Lecce, Italy \\ E-mail: florio@farmchim.uniba.it; icho-s@icho.edu.pl
}

\section{Dedicated to Professor Giuseppe Bartoli on the occasion of his 65th Anniversary}

\begin{abstract}
The carbanion of 4,4-dimethyl-2-(1-phenylethyl)-4,5-dihydro-1,3-oxazole adds 1 to nitroarenes in the position para to the nitro group: oxidation of the produced $\sigma^{\mathrm{H}}$ adducts with dichlorodicyanobenzoquinone (DDQ) gives para-substituted nitroarenes whereas oxidation with dimethyldioxirane (DMD) leads to para-substituted phenols.
\end{abstract}

Keywords: Carbanions, nitroarenes, VNS reaction, ONSH reaction, oxazolines, phenols

\section{Introduction}

Nucleophilic substitution of hydrogen in nitroarenes and other electron-deficient arenes is a well recognized process of substantial value in organic synthesis. ${ }^{1,2}$ There are two main variants of this process: vicarious and oxidative nucleophilic substitution. The key step of both of these reactions is the formation of $\sigma^{\mathrm{H}}$ adducts via addition of nucleophiles to the electron-deficient aromatic rings in positions occupied with hydrogen. When the nucleophiles contain leaving groups L at the nucleophilic center, e.g., $\alpha$-halocarbanions or alkyl hydroperoxide anions further transformation of the $\sigma^{\mathrm{H}}$ adducts proceeds with base-induced $\beta$-elimination of HL giving products of vicarious nucleophilic substitution (VNS). ${ }^{3,4}$ On the other hand, oxidation of the $\sigma^{\mathrm{H}}$ adducts with external oxidants results in oxidative nucleophilic substitution of hydrogen $(\mathrm{ONSH}) .{ }^{5}$ Of particular value in organic synthesis is the nucleophilic oxidative alkylation of nitroarenes with Grignard reagents, pioneered by Bartoli. ${ }^{6,7}$ He has shown that alkyl Grignard reagents add to the electron-deficient rings of nitroarenes, while subsequent oxidation of the 
produced $\sigma^{\mathrm{H}}$ adducts with a variety of oxidants gives the alkylation products. Recently, it has been found that the most efficient reagent for oxidation of these $\sigma^{\mathrm{H}}$ adducts is $\mathrm{KMnO}_{4}$ in liquid ammonia. ${ }^{8}$ However, the ONSH reaction in nitroarenes with stabilized carbanions is somewhat limited owing to the reversibility of the $\sigma^{\mathrm{H}}$ adducts formation. Nevertheless, it serves as a valuable tool for the introduction of functionalized alkyl substituents into aromatic rings. ${ }^{5,9}$

Oxazolines have become well established as highly versatile compounds in organic synthesis. The heterocyclic system serves as a latent function that can be transformed into a variety of other functional groups, while most of the chemical manipulations involve the development of substituents borne at the 2-position. One of the key features of the oxazoline system is the stabilization of carbanions generated at the $\alpha$-center of a 2-alkyl derivative and subsequent reactions of such carbanions with a variety of electrophilic reagents. ${ }^{10 a}$ Following the pioneering work of Meyers, ${ }^{1 \mathrm{a}}$ this reactivity has been exploited extensively in asymmetric synthesis. ${ }^{10 \mathrm{~b}, \mathrm{c}}$ Metallated $\alpha$-hetero-substituted 2-alkyloxazolines have been investigated extensively in the laboratory of one of the authors, as well as the synthetic applications. ${ }^{11}$

Recently, we have reported that carbanions of $\alpha$-chloroalkyl- and dichloromethyl- oxazolines undergo the VNS reaction with a variety of nitroarenes, producing nitrobenzyloxazolines ${ }^{12}$ and $\alpha$-chloronitrobenzyl oxazolines. ${ }^{13}$ The latter compounds can enter in situ the Darzens reaction with aldehydes giving nitroaryl oxazolinyl oxiranes. ${ }^{13}$

In order to extend the scope of the methods for introduction of alkyloxazolinyl substituents into nitro-aromatic rings we have studied ONSH in nitroarenes with a carbanion stabilized by the oxazolinyl group. The nature of the carbanion-stabilizing group that decides the nucleophilicity of the carbanion, and hence the addition equilibrium and orientation of the addition due to steric effect, is of crucial importance in the reactions of the carbanion with nitroarenes.

\section{Results and Discussion}

As a model carbanion precursor for the ONSH reaction we have chosen 4,4-dimethyl-2-(1phenylethyl)-4,5-dihydro-1,3-oxazoline 1, readily prepared from 2-phenylpropionic acid and 2amino-2-methyl propanol. ${ }^{14}$ The phenyl group in $\mathbf{1}$ provides additional stabilization to the carbanion, assuring facile deprotonation of $\mathbf{1}$ and proper nucleophilicity of the carbanion. Due to steric effects of the large oxazolinyl moiety and the methyl and phenyl groups, addition of the tertiary carbanion of $\mathbf{1}$ to nitroarenes should proceed exclusively in the para position, whereas products of the $\mathrm{ONSH}$ reaction are not $\mathrm{CH}$ acids so they should be stable in the reaction medium in the presence of bases and oxidants. In some preliminary experimentation we found that the ONSH reaction of $\mathbf{1}$ proceeds efficiently when the carbanion is generated upon treatment of $\mathbf{1}$ with $t$-BuOK in THF at low temperature in the presence of the nitroarene, and the produced $\sigma^{\mathrm{H}}$ adducts are subsequently oxidized by dichlorodicyanobenzoquinone (DDQ) to give the nitroarene containing an alkyloxazoline substituent para to the nitro group (Scheme 1a). 


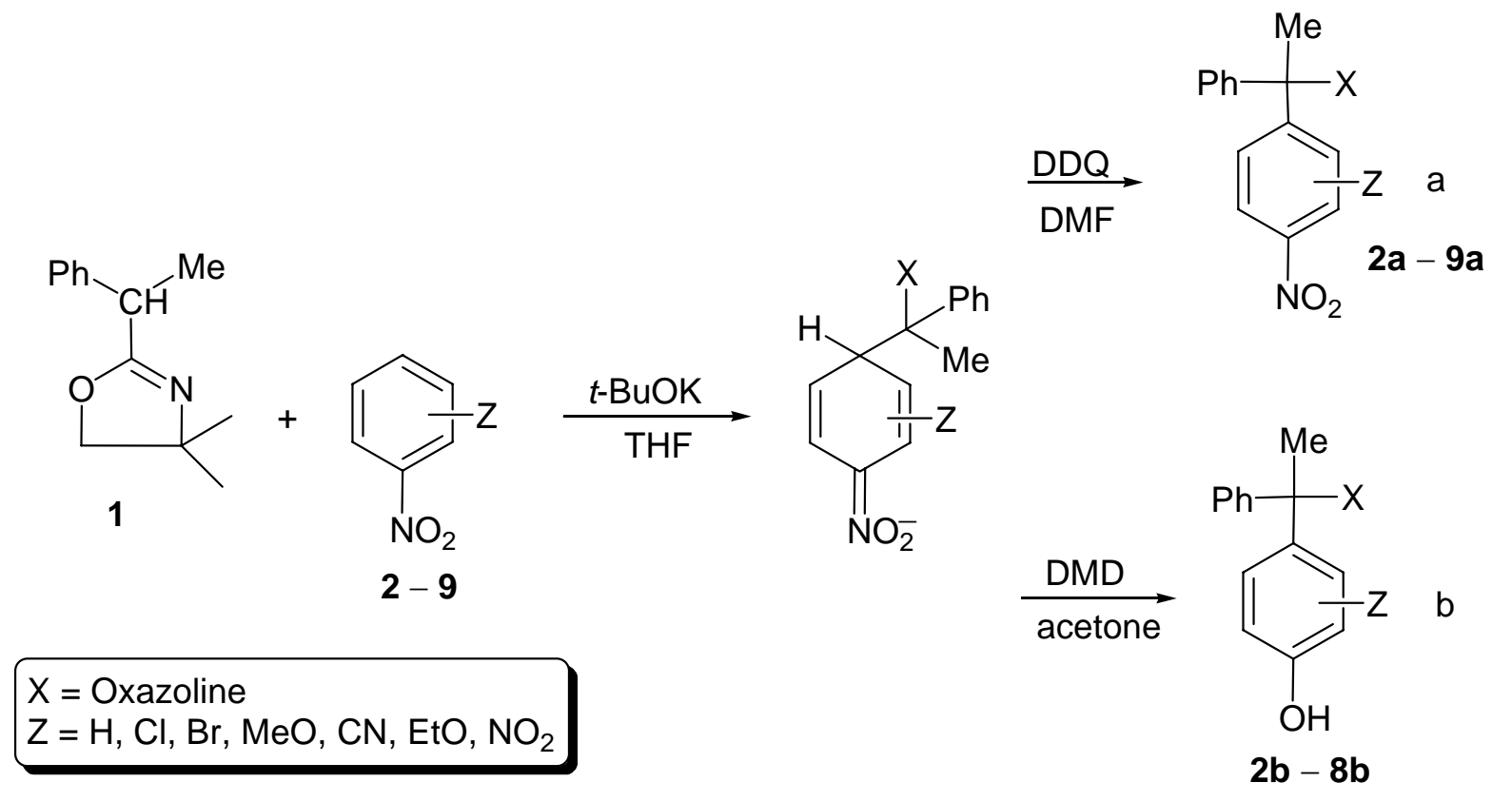

\section{Scheme 1}

The results presented in Table 1 show that the ONSH reaction proceeds satisfactorily under these conditions, giving the expected products of para substitution in moderate to excellent yields. Another efficient base solvent system for the ONSH reaction with carbanions is $\mathrm{NaNH}_{2}$ in liquid ammonia with $\mathrm{KMnO}_{4}$ as the oxidant. ${ }^{15}$ We have found that this system can also be applied for the reaction of $\mathbf{1}$. Treatment of $\mathbf{1}$ with $\mathrm{NaNH}_{2}$ in liquid ammonia and subsequently with nitrobenzene and $\mathrm{KMnO}_{4}$ gave $2 \mathrm{a}$ in an excellent yield (90\%).

Anionic $\sigma^{\mathrm{H}}$ adducts of the addition of carbanions to nitroarenes can be oxidized also by dimethyldioxirane (DMD): however, this oxidizes the negatively charged nitro groups, so that the final products are substituted phenols. ${ }^{16}$ This oxidant can also be applied to the oxidation of the $\sigma^{\mathrm{H}}$ adducts derived from the oxazoline-stabilized carbanion of $\mathbf{1}$ and nitroarenes. A series of phenols containing alkyl oxazolinyl substituents in the para position was obtained by this way (Scheme 1b).

\section{Conclusions}

We have shown that the oxidative nucleophilic substitution of hydrogen in nitroarenes with the stabilized carbanion derived from 4,4-dimethyl-2-(1-phenylethyl)-4,5-dihydro-1,3-oxazole 1 proceeds efficiently. Oxidation of the intermediate $\sigma^{\mathrm{H}}$ adducts derived from this carbanion and nitroarenes with DDQ and DMD permits the preparation of para-nitrobenzyl- and parahydroxybenzyl- oxazolines respectively, which are valuable intermediates in organic synthesis. 
Table 1. Oxidation of $\sigma^{\mathrm{H}}$ adducts of $\mathbf{1}^{-}$to nitroarenes by DDQ and DMD according to Scheme 1

\begin{tabular}{ccccccc}
\hline & & \multicolumn{5}{c}{ Oxidant } \\
\hline Entry & $\mathbf{Z}$ & No. & \multicolumn{2}{c}{ DDQ } & \multicolumn{2}{c}{ DMD } \\
\hline & & & product & Yield \% & Product & Yield \% \\
\hline 1 & $\mathrm{H}$ & $\mathbf{2}$ & $\mathbf{2 a}$ & 95 & $\mathbf{2 b}$ & 53 \\
2 & $3-\mathrm{Cl}$ & $\mathbf{3}$ & $\mathbf{3 a}$ & 90 & $\mathbf{3 b}$ & 74 \\
3 & $2-\mathrm{Cl}$ & $\mathbf{4}$ & $\mathbf{4 a}$ & 78 & & \\
4 & $3-\mathrm{Br}$ & $\mathbf{5}$ & $\mathbf{5 a}$ & 74 & $\mathbf{5 b}$ & 68 \\
5 & $2-\mathrm{Br}$ & $\mathbf{6}$ & $\mathbf{6 a}$ & 74 & & \\
6 & $2-\mathrm{OMe}$ & $\mathbf{7}$ & $\mathbf{7 a}$ & 92 & & \\
7 & $3-\mathrm{CN}$ & $\mathbf{8}$ & $\mathbf{8 a}$ & 58 & $\mathbf{8 b}$ & 35 \\
8 & $4-\mathrm{EtO}-3-$ & $\mathbf{9}$ & $\mathbf{9 a}$ & 63 & & \\
\hline
\end{tabular}

\section{Experimental Section}

General Procedures. Melting points were uncorrected. ${ }^{1} \mathrm{H}$ NMR spectra were recorded in $\mathrm{CDCl}_{3}$ on $200 \mathrm{MHz}$ or $400 \mathrm{MHz}$ instruments. Mass spectra were measured on an AMD 604 Inectra GmbH spectrometer using EI. For column chromatography, silica gel (230-400 mesh), Merck, was used. DMF was distilled over calcium hydride and stored over molecular sieves; tetrahydrofuran was distilled over potassium benzophenone ketyl. Potassium tert-butoxide was reagent grade purchased from Fluka. An acetone solution of DMD was prepared according to the procedure described by Adam et al. ${ }^{17}$ All reactions were performed under an argon atmosphere.

The starting nitroarenes were commercial products. 4,4-dimethyl-2-(1-phenylethyl)-4,5dihydro-1,3-oxazole 1 was prepared starting from 2-phenylpropionic acid following a procedure reported in ref. 14.

In a 100-ml round bottom flask, 2-phenylpropionic acid (100 $\mathrm{mmol})$ and 2-amino-2-methylpropanol $(100 \mathrm{mmol})$ were added in $50 \mathrm{ml}$ of xylene. The flask was connected with a Dean-Stark apparatus, warmed up to $150^{\circ} \mathrm{C}$, and heated under reflux for about $15 \mathrm{~h}$, until no more water was collected. A solution of sodium bicarbonate $\left(40 \mathrm{ml}, \mathrm{NaHCO}_{3} 10 \%\right)$ was added, and the mixture extracted with methylene chloride $(3 \times 20 \mathrm{ml})$. The solvent was evaporated and the product, identified as 2-(1-Phenylethyl)-4,4-dimethyl-2-oxazoline (1), was purified by distillation $\left(127^{\circ} \mathrm{C}\right.$ at $0.1 \mathrm{mbar})$. Colorless oil, yield 70\%. ${ }^{1} \mathrm{H}$ NMR $(200 \mathrm{MHz}) \delta 7.28-7.38(\mathrm{~m}, 5 \mathrm{H}), 3.92-3.85(\mathrm{~m}$, $2 \mathrm{H}), 3.71(\mathrm{q}, J=7.1 \mathrm{~Hz}, 1 \mathrm{H}), 1.54(\mathrm{~d}, J=7.1 \mathrm{~Hz}, 3 \mathrm{H}), 1.30(\mathrm{~m}, 6 \mathrm{H}) ;{ }^{13} \mathrm{C} \mathrm{NMR}(50 \mathrm{MHz}$, $\left.\mathrm{CDCl}_{3}\right) \delta 167.5,141.6,128.5,127.1,126.2,79.0,66.7,39.4,28.3,28.1$; GC-MS (70 eV) m/z (\%): $203\left(\mathrm{M}^{+}, 100\right), 188$ (98), 126 (60), 105 (97), 91 (57), 77 (73); FT-IR (film, cm ${ }^{-1}$ ): 2950, $1662(\mathrm{~s}, \mathrm{C}=\mathrm{N}), 1430,1250,1145$. 
Procedure for the oxidative substitution of hydrogen with 4,4-dimethyl-2-(1-phenylethyl)4,5-dihydro-1,3-oxazole (1) with t-BuOK in nitroarenes and DDQ. To a stirred solution of $t$ $\mathrm{BuOK}(2.5 \mathrm{mmol})$ in a mixture of THF and DMF $(50: 50)$ at $-70^{\circ} \mathrm{C}$, a solution of nitroarene $(1.05$ $\mathrm{mmol})$ and oxazoline 1 (1 mmol) in THF (1 ml) was added. After 15 minutes a solution of DDQ $(1.2 \mathrm{mmol})$ in DMF $(1 \mathrm{ml})$ was added at $-70^{\circ} \mathrm{C}$. The reaction mixture was stirred at this temperature for 10 minutes, treated with an aqueous solution of ammonium chloride and the cooling bath was removed. The crude material was extracted with methylene chloride (3x20 ml). The solvent was evaporated and the products purified by column chromatography using hexaneethyl acetate 5:1 as eluent, or recrystallized from $n$-heptane.

Oxidative substitution of hydrogen with 4,4-dimethyl-2-(1-phenylethyl)-4,5-dihydro-1,3oxazole (1) and t-BuOK in nitroarenes and DMD. To a stirred solution of $t$-BuOK (2.5 mmol) in THF at $-70^{\circ} \mathrm{C}$ a solution of oxazoline $1(1 \mathrm{mmol})$ in $0.5 \mathrm{ml}$ of DMF was added. After $3 \mathrm{~min}$ a solution of the nitroarene $(1.2 \mathrm{mmol})$ in THF was added, and the mixture stirred at this temperature for $15 \mathrm{~min}$; water $(18 \mu \mathrm{l}, 1.2 \mathrm{mmol})$ and then an acetone solution of DMD (ca. 1.2 mmol, $20 \mathrm{ml}$ of $c a .0 .06 \mathrm{M}$ ) was added to the mixture. After 10 minutes, aqueous ammonium chloride was added and the cooling bath was removed. The crude product was extracted with methylene chloride ( $3 \times 20 \mathrm{ml}$ ), the solvent evaporated, and the phenols purified from traces of the corresponding nitro- derivatives by column chromatography using hexane-ethyl acetate, 7:3, as eluent.

4,4-Dimethyl-2-[1-(4-nitrophenyl)-1-phenylethyl]-4,5-dihydro-oxazole (2a). Yellow crystals, m. p. 79-80 ${ }^{\circ} \mathrm{C}$ (n-heptane), yield 95\%. ${ }^{1} \mathrm{H}$ NMR (400 MHz) $\delta$ 8.18-8.10 (m, $\left.2 \mathrm{H}\right)$, 7.47-7.39 (m, $2 \mathrm{H}), 7.38-7.27$ (m, $3 \mathrm{H})$, 7.27-7.20 (m, $2 \mathrm{H}), 4.02-3.90(\mathrm{~m}, 2 \mathrm{H}), 2.02$ (s, $3 \mathrm{H}), 1.36(\mathrm{~s}, 3 \mathrm{H}), 1.29$ $(\mathrm{s}, 3 \mathrm{H}) .{ }^{13} \mathrm{C}$ NMR $(100 \mathrm{MHz}) \delta 167.8,152.6,146.6,143.4,129.1,128.4,127.6,127.3,123.1$, 79.4, 67.3, 50.4, 28.1, 27.9, 24.0. EI MS m/z (\%): 324 (70), 323 (100), 294 (3), 226 (14), 178 (11). Anal. Calcd for $\mathrm{C}_{19} \mathrm{H}_{20} \mathrm{~N}_{2} \mathrm{O}_{3}(\mathrm{M}=324.38)$ : C, 70.35; H, 6.38, N 8.64; Found: $\mathrm{C} 70.45$; H, $6.38 ; \mathrm{N}, 8.49 \%$. HR MS (EI): Calc. for $\mathrm{C}_{19} \mathrm{H}_{20} \mathrm{~N}_{2} \mathrm{O}_{3} \mathrm{M}=324.1474$; Found $\mathrm{M}=324.1468$.

2-[1-(2-Chloro-4-nitrophenyl)-1-phenylethyl]-4,4-dimethyl-4,5-dihydro-1,3-oxazole (3a). Yellow crystals, m. p. $103-104^{\circ} \mathrm{C}$ (n-heptane), yield 90\%. ${ }^{1} \mathrm{H}$ NMR (400 MHz) $\delta 8.23$ (d, $1 \mathrm{H}, J$ $=2.3 \mathrm{~Hz}), 7.90(\mathrm{dd}, 1 \mathrm{H}, J=8.8,2.3 \mathrm{~Hz}), 7.54-7.47(\mathrm{~m}, 2 \mathrm{H}), 7.44-7.34(\mathrm{~m}, 3 \mathrm{H}), 6.95(\mathrm{~d}, 1 \mathrm{H}, J$ $=8.8 \mathrm{~Hz}), 4.02-3.95(\mathrm{~m}, 2 \mathrm{H}), 2.12(\mathrm{~s}, 3 \mathrm{H}), 1.34(\mathrm{~s}, 3 \mathrm{H}), 1.20(\mathrm{~s}, 3 \mathrm{H}) .{ }^{13} \mathrm{C} \mathrm{NMR}(100 \mathrm{MHz}) \delta$ $166.9,150.5,146.8,141.5,135.1,130.7,128.6,127.7,127.2,126.2,121.3,70.5,67.4,50.3,27.9$, 27.8, 23.8. Anal. Calcd. for $\mathrm{C}_{19} \mathrm{H}_{19} \mathrm{ClN}_{2} \mathrm{O}_{3}$ (358.82): C, 63.60; H, 5.34; Cl, 9.88; N, 7.81. Found: C, 63.71; H, 5.21; Cl, 9.62; N, 7.80.

2-[1-(3-Chloro-4-nitrophenyl)-1-phenylethyl]-4,4-dimethyl-4,5-dihydro-1,3-oxazole (4a). Yellow oil, yield 78\%. ${ }^{1} \mathrm{H}$ NMR (400 MHz) $\delta 7.81(\mathrm{~d}, 1 \mathrm{H}, \mathrm{J}=8.6 \mathrm{~Hz}), 7.43$ (d, $1 \mathrm{H}, \mathrm{J}=2.0 \mathrm{~Hz}$ ), 7.39-7.20 (m, $6 \mathrm{H}), 4.16-4.07(\mathrm{~m}, 2 \mathrm{H}), 1.96(\mathrm{~s}, 3 \mathrm{H}), 1.33(\mathrm{~s}, 3 \mathrm{H}), 1.31(\mathrm{~s}, 3 \mathrm{H}) .{ }^{13} \mathrm{C}$ NMR $(100$ $\left.\mathrm{MHz}, \mathrm{CDCl}_{3}\right) \delta 167.3,151.6,146.1,142.9,131.6,128.5,127.6,127.5,127.4,127.2,125.2,79.5$, 67.4, 50.1, 28.2, 28.1, 27.9. HR MS (EI): HRMS (EI): Calcd. for $\mathrm{C}_{19} \mathrm{H}_{19}{ }^{35} \mathrm{ClN}_{2} \mathrm{O}_{3}$ : 358.1084 . Found: 358.1091. 
2-[1-(2-Bromo-4-nitrophenyl)-1-phenylethyl]-4,4-dimethyl-4,5-dihydro-1,3-oxazole

(5a).

Yellow crystals, m. p. $114-115^{\circ} \mathrm{C}$ (n-heptane), yield 74\%. ${ }^{1} \mathrm{H}$ NMR $(400 \mathrm{MHz}) \delta 8.44(\mathrm{~d}, 1 \mathrm{H}, \mathrm{J}$ $=2.4 \mathrm{~Hz}), 7.96(\mathrm{dd}, 1 \mathrm{H}, J=8.8$ and $2.4 \mathrm{~Hz}), 7.55-7.50(\mathrm{~m}, 2 \mathrm{H}), 7.40-7.30(\mathrm{~m}, 3 \mathrm{H}), 6.99(\mathrm{~d}, 1$

$\mathrm{H}, J=8.8 \mathrm{~Hz}), 4.03-3.97(\mathrm{~m}, 2 \mathrm{H}), 2.15(\mathrm{~s}, 3 \mathrm{H}), 1.35(\mathrm{~s}, 3 \mathrm{H}), 1.20(\mathrm{~s}, 3 \mathrm{H}) .{ }^{13} \mathrm{C}$ NMR $(100$ $\mathrm{MHz}) \delta 166.7,151.8,146.5,141.6,130.8,129.7,128.5,128.2,127.7,124.1,121.7,79.4,67.4$, 51.4, 27.8, 27.7, 24.1. Anal. Calcd. for $\mathrm{C}_{19} \mathrm{H}_{19} \mathrm{BrN}_{2} \mathrm{O}_{3}$ (403.27): C, 56.59; H, 4.75; Br, 19.81; N, 6.95. Found: C, 56.46; H, 4.70; Br, 19.86; N, 7.26. HRMS (EI): Calcd. for $\mathrm{C}_{19} \mathrm{H}_{19}{ }^{79} \mathrm{BrN}_{2} \mathrm{O}_{3}$ : 402.0579. Found: 402.0571. MS EI m/z (\%): 403 (64), 402 (50), 323 (100), 304 (16), 203 (20), 178 (19), 105 (100).

2-[1-(3-Bromo-4-nitrophenyl)-1-phenylethyl]-4,4-dimethyl-4,5-dihydro-1,3-oxazole

(6a). Yellow oil, yield 74\%. ${ }^{1} \mathrm{H}$ NMR (400 MHz) $\delta 7.78(\mathrm{~d}, 1 \mathrm{H}, J=8.5 \mathrm{~Hz}), 7.62(\mathrm{~d}, 1 \mathrm{H}, J=2.1 \mathrm{~Hz})$, 7.38-7.20 (m, 6H), 4.00-3.90 (m, 2H), $1.96(\mathrm{~s}, 3 \mathrm{H}), 1.33$ (s, 3H), 1.31 (s, 3H). ${ }^{13} \mathrm{C}$ NMR (100 MHz) $\delta 167.3,151.5,148.0,142.9,134.7,128.5,128.3,127.5,127.4,125.2,114.2,79.5,67.4$, 50.0, 28.3, 28.1. HR MS (EI): Calc. for $\mathrm{C}_{19} \mathrm{H}_{20} \mathrm{~N}_{2} \mathrm{O}_{3}{ }^{79} \mathrm{Br} \mathrm{M}+\mathrm{H}^{+}=403.0652$; Found $\mathrm{M}=$ 403.0668. MS EI m/z (\%): 403 (100), 402 (70), 323 (70), 304 (20), 178 (35).

2-[1-(3-Methoxy-4-nitrophenyl)-1-phenylethyl]-4,4-dimethyl-4,5-dihydro-1,3-oxazole (7a). Yellow oil, yield 92\%. ${ }^{1} \mathrm{H}$ NMR (400 MHz) $\delta 7.80(\mathrm{~d}, 1 \mathrm{H}, J=8.6 \mathrm{~Hz}), 7.39-7.20$ (m, $\left.5 \mathrm{H}\right), 7.01$ $(\mathrm{d}, 1 \mathrm{H}, J=1.9 \mathrm{~Hz}), 6.94(\mathrm{dd}, 1 \mathrm{H}, \mathrm{J}=8.6$ and $1.9 \mathrm{~Hz}), 4.00-3.91(\mathrm{~m}, 2 \mathrm{H}), 3.83(\mathrm{~s}, 3 \mathrm{H}), 1.97$ (s, $3 \mathrm{H}), 1.33(\mathrm{~s}, 3 \mathrm{H}), 1.32(\mathrm{~s}, 3 \mathrm{H}) .{ }^{13} \mathrm{C} \mathrm{NMR}(100 \mathrm{MHz}) \delta 167.9,152.5,152.3,143.4,137.9$, $128.3,127.7,127.3,125.3,120.0,113.8,79.4,67.3,56.3,50.5,28.1,28.0,23.0$. HR MS (EI): Calc. for $\mathrm{C}_{20} \mathrm{H}_{22} \mathrm{~N}_{2} \mathrm{O}_{4}, \mathrm{M}=354.1579$; Found $\mathrm{M}=354.1567$.

2-[1-(4,4-Dimethyl-4,5-dihydro-1,3-oxazol-2-yl)-1-phenylethyl]-5-nitrobenzenecarbonitrile

(8a). Yellow crystals, m. p. $155-157^{\circ} \mathrm{C}$ (n-heptane), yield $58 \% .{ }^{1} \mathrm{H}$ NMR $(400 \mathrm{MHz}) \delta 8.52(\mathrm{~d}, 1$ $\mathrm{H}, J=2.5 \mathrm{~Hz}), 8.17(\mathrm{dd}, 1 \mathrm{H}, J=8.8$ and $2.5 \mathrm{~Hz}), 7.51-7.40(\mathrm{~m}, 5 \mathrm{H}), 7.00(\mathrm{~d}, 1 \mathrm{H}, J=8.8 \mathrm{~Hz})$, 4.16-4.00 (m, $2 \mathrm{H}), 2.17(\mathrm{~s}, 3 \mathrm{H}), 1.36(\mathrm{~s}, 3 \mathrm{H}), 1.31(\mathrm{~s}, 3 \mathrm{H}) \cdot{ }^{13} \mathrm{C}$ NMR $(100 \mathrm{MHz}) \delta 172.1$, 156.1, 146.1, 140.3, 130.1, 129.5, 128.9, 128.3, 127.6, 126.7, 116.8, 114.5, 79.8, 67.9, 50.3, 27.9, 27.5, 25.0. Anal. Calcd. for $\mathrm{C}_{20} \mathrm{H}_{19} \mathrm{~N}_{3} \mathrm{O}_{3}$ (349.39): C, 68.75; H, 5.48; N, 12.03. Found: C, 68.53; $\mathrm{H}, 5.27$; N, 11.78 .

2-[1-(4,4-Dimethyl-4,5-dihydro-1,3-oxazol-2-yl)-1-phenylethyl]-4-ethoxy-5-nitropyridine

(9a). Colorless crystals, m. p. 92-93 ${ }^{\circ} \mathrm{C}$ (n-heptane), yield 63\%. ${ }^{1} \mathrm{H}$ NMR (400 MHz) $\delta 8.96$ (s, 1 H), 7.39-7.28 (m, $5 \mathrm{H}), 6.91(\mathrm{~s}, 1 \mathrm{H}), 4.03(\mathrm{dq}, 2 \mathrm{H}, J=7.0$ and 1.5 Hz), 4.00-3.91 (m, $2 \mathrm{H}), 2.04$ $(\mathrm{s}, 3 \mathrm{H}), 1.39$ (t, $3 \mathrm{H}, J=7.0 \mathrm{~Hz}), 1.34(\mathrm{~s}, 3 \mathrm{H}), 1.32(\mathrm{~s}, 3 \mathrm{H}) \cdot{ }^{13} \mathrm{C}$ NMR $(100 \mathrm{MHz}): \delta 169.7$, 167.5, 158.0, 145.9, 143.1, 135.1, 128.4, 127.5, 127.3, 108.9, 79.4, 67.3, 65.3, 53.2, 28.1, 26.5, 14.0. Anal. Calcd. for $\mathrm{C}_{20} \mathrm{H}_{23} \mathrm{~N}_{3} \mathrm{O}_{4}$ (369.42): C, 65.03; H, 6.28; N, 11.37. Found: C, 65.12; H, 6.40; N, 11.27. HRMS (EI): Calcd. for $\mathrm{C}_{20} \mathrm{H}_{23} \mathrm{~N}_{3} \mathrm{O}_{4}$ : 369.16899. Found: 369.1700 .

4-[1-(4,4-Dimethyl-4,5-dihydro-1,3-oxazol-2-yl)-1-phenylethyl]phenol (2b). Colorless crystals, m. p. $207-209^{\circ} \mathrm{C}$ (ethanol), yield 53\%. ${ }^{1} \mathrm{H}$ NMR $(200 \mathrm{MHz}) \delta 9.92$ (s, $1 \mathrm{H}$, broad), 7.427.29 (m, 5 H), 7.11-7.01 (m, 2 H), 6.69-6.61 (m, 2 H), 4.09-4.00 (m, 2 H), 2.02 (s, 3 H), 1.43 (s, $3 \mathrm{H}), 1.41$ (s, $3 \mathrm{H}) .{ }^{13} \mathrm{C}$ NMR $(50 \mathrm{MHz}) \delta 170.5,155.0,144.8,135.9,128.9,128.1,127.5,126.7$, 
115.2, 79.4, 67.0, 49.5, 28.2, 28.1, 27.8. Anal. Calcd. for $\mathrm{C}_{19} \mathrm{H}_{21} \mathrm{NO}_{2}$ (295.38): C, 77.26; H, 7.17; N, 4.74. Found: C, 77.26; H, 7.32; N, 4.75.

3-Chloro-4-[1-(4,4-dimethyl-4,5-dihydro-1,3-oxazol-2-yl)-1-phenylethyl]phenol (3b). Colorless crystals, m. p. $213-215^{\circ} \mathrm{C}$ (ethanol), yield 74\%. ${ }^{1} \mathrm{H}$ NMR $(200 \mathrm{MHz}) \delta 10.26$ (s, $1 \mathrm{H}$, broad), 7.58-7.48 (m, $2 \mathrm{H}), 7.46-7.30(\mathrm{~m}, 3 \mathrm{H}), 6.68(\mathrm{~d}, 1 \mathrm{H}, J=2.5 \mathrm{~Hz}), 6.38(\mathrm{~d}, 1 \mathrm{H}, J=8.8 \mathrm{~Hz}), 6.26$ $(\mathrm{dd}, 1 \mathrm{H}, J=8.8$ and $2.5 \mathrm{~Hz}), 4.21-4.00(\mathrm{~m}, 2 \mathrm{H}), 2.09(\mathrm{~s}, 3 \mathrm{H}), 1.51(\mathrm{~s}, 3 \mathrm{H}), 1.37(\mathrm{~s}, 3 \mathrm{H}) .{ }^{13} \mathrm{C}$ NMR $(50 \mathrm{MHz}) \delta 171.0,156.7,141.9,133.0,132.6,129.9,128.3,128.0,127.2,119.1,114.2$, 79.7, 67.1, 49.3, 31.8, 27.9, 27.8. Calc. for $\mathrm{C}_{19} \mathrm{H}_{20} \mathrm{NO}_{2} \mathrm{Cl}(\mathrm{M}=329.83): \mathrm{C}, 69.19 ; \mathrm{H}, 6.11 ; \mathrm{N}$ 4.25; Cl, 10.75. Found: C 69.33; H, 6.29; N, 4.43; Cl, 10.45 .

3-Bromo-4-[1-(4,4-dimethyl-4,5-dihydro-1,3-oxazol-2-yl)-1-phenylethyl]phenol (5b). Colorless crystals, m. p. $208-209^{\circ} \mathrm{C}$ (ethanol), yield 68\%. ${ }^{1} \mathrm{H}$ NMR $(200 \mathrm{MHz}) \delta 10.10$ (s, $1 \mathrm{H}$, broad), 7.55-7.49 (m, $2 \mathrm{H}), 7.41-7.30$ (m, $3 \mathrm{H}), 6.94$ (d, $1 \mathrm{H}, J=2.5 \mathrm{~Hz}), 6.46(\mathrm{~d}, 1 \mathrm{H}, J=8.8 \mathrm{~Hz}), 6.34$ $(\mathrm{dd}, 1 \mathrm{H}, J=8.8$ and $2.5 \mathrm{~Hz}), 4.18-4.00(\mathrm{~m}, 2 \mathrm{H}), 2.11(\mathrm{~s}, 3 \mathrm{H}), 1.51(\mathrm{~s}, 3 \mathrm{H}), 1.38(\mathrm{~s}, 3 \mathrm{H}) .{ }^{13} \mathrm{C}$ NMR $(50 \mathrm{MHz}) \delta 170.8,156.8,141.8,133.6,130.3,128.4,128.3,127.4,123.5,122.4,114.8$, 80.0, 66.8, 50.5, 27.7, 27.6. 25.3. Anal. Calcd. for $\mathrm{C}_{19} \mathrm{H}_{20} \mathrm{BrNO}_{2}$ (374.28): C, 60.97; H, 5.39; $\mathrm{Br}$, 21.35; N, 3.74. Found: C, 61.18; H, 5.20; Br, 21.24; N, 3.69.

2-[1-(4,4-Dimethyl-4,5-dihydro-oxazol-2-yl)-1-phenylethyl]-5-hydroxy-benzonitrile

(8b). Colorless crystals, m. p. $231-232^{\circ} \mathrm{C}$ (ethanol), yield 35\%. ${ }^{1} \mathrm{H}$ NMR (400 MHz) $\delta 10.23(\mathrm{~s}, 1 \mathrm{H}$, broad), 7.52-7.35 (m, $5 \mathrm{H}), 6.73(\mathrm{~d}, 1 \mathrm{H}, J=2.7 \mathrm{~Hz}), 6.56(\mathrm{dd}, 1 \mathrm{H}, \mathrm{J}=8.8$ and $2.7 \mathrm{~Hz}), 6.35(\mathrm{~d}$, $1 \mathrm{H}, J=8.8 \mathrm{~Hz}), 4.25(\mathrm{~d}, 1 \mathrm{H}, J=8.25 \mathrm{~Hz}), 4.06(\mathrm{~d}, 1 \mathrm{H}, J=8.25 \mathrm{~Hz}), 2.13(\mathrm{~s}, 3 \mathrm{H}), 1.59$ (s, 3 $\mathrm{H}), 1.36(\mathrm{~s}, 3 \mathrm{H}) .{ }^{13} \mathrm{C} \mathrm{NMR}(100 \mathrm{MHz}) \delta 171.2,155.8,140.7,139.0,129.8,128.8,127.9,127.6$, 122.1, 120.2, 119.2, 112.2, 79.9, 67.4, 49.4, 27.9, 27.5, 25.0. Anal. Calcd. for $\mathrm{C}_{20} \mathrm{H}_{20} \mathrm{~N}_{2} \mathrm{O}_{2}$ (320.39): C, 74.98; H, 6.29; N, 8.74. Found: C, 74.84; H, 6.32; N, 8.41.

\section{Acknowledgements}

The authors are grateful for financial support by the Ministero dell'Istruzione, dell'Università e della Ricerca (MIUR, Rome) under the framework of the National Project, "Stereoselezione in Sintesi Organica, Metodologie ed Applicazioni" and the FIRB Project, "Progettazione, preparazione e valutazione biologica e farmacologica di nuove molecole organiche quali potenziali farmaci innovative", by the Universities of Bari, Lecce (Italy) and Warszawa (Poland).

\section{References}

1. Mąkosza, M.; Wojciechowski, K. Chem. Rev. 2004, 104, 2631.

2. (a) Mąkosza, M. Russ. Chem. Bull. 1996, 45, 491. (b) Chupakhin, O.N.; Charushin, V. N.; van der Plas H. C. Nucleophilic Aromatic Substitution of Hydrogen, Academic Press: San 
Diego 1994. Terrier, F. Nucleophilic Aromatic Displacement, VCH Publishers: Weinheim, 1991.

3. (a) Mąkosza, M.; Winiarski, J. Acc. Chem. Res. 1987, 20, 282. (b) Mąkosza, M.; Kwast, A. J. Phys. Org. Chem. 1998, 11, 341.

4. Mąkosza, M.; Wojciechowski, K. Liebigs Annalen Receuil 1997, 1805.

5. (a) Mąkosza, M.; Staliński, K. Polish J. Chem. 1999, 73, 151. (b) Mąkosza, M.; Paszewski, M. Polish J. Chem. 2005, 79, 163.

6. Bartoli, G. Acc. Chem. Res. 1984, 17, 109.

7. Bartoli, G.; Bosco, M. J. Org. Chem. 1980, 45, 522; 1982, 47, 5227.

8. Mąkosza, M.; Surowiec, M. J. Organomet. Chem. 2001, 624, 167.

9. RajanBabu, T.V.; Fukunaga, T. J. Org. Chem. 1984, 49, 4571.

10. (a) Reuman, M.; Meyers, A. I. Tetrahedron 1985, 41, 837. (b) Meyers, A. I. J. Heterocyclic Chem. 1998, 35, 989. (c) Meyers, A. I. J. Org. Chem. 2005, 70, 6137.

11. (a) Capriati, V.; Florio, S.; Luisi, R. Synlett. 2005, 1359. (b) Luisi, R.; Capriati, V.; Florio, S.; Di Cunto, P.; Musio, B. Tetrahedron 2005, 61, 3251. (c) Capriati, V.; Florio, S.; Luisi, R. Curr. Org. Chem. 2004, 8, 1529. (d) Florio, S.; Perna, F. M.; Luisi, R.; Barluenga, J.; Fananas, F. J.; Rodriguez, F. J. Org. Chem. 2004, 69, 9204. (e) Florio, S.; Perna, F. M.; Luisi, R.; Barluenga, J.; Fananas, F. J.; Rodriguez, F. J. Org. Chem. 2004, 69, 6480. (f) Abbotto, A.; Capriati, V.; Degennaro, L.; Florio, S.; Luisi, R.; Pierrot, M., Salomone, A. J. Org. Chem. 2001, 66, 3049. (g) Luisi, R.; Capriati, V.; Florio, S.; Vista, T. J. Org. Chem. 2003, 68. 9861. (h) Luisi, R.; Capriati, V.; Florio, S.; Piccolo, E. J. Org. Chem. 2003, 68, 10187. Luisi, R.; Capriati, V.; Degennaro, L.; Florio, S. Org. Lett. 2003, 5, 2723. (i) Capriati, V.; Degennaro, L.; Favia, R.; Florio, S. Org. Lett. 2002, 4, 2445.

12. Florio, S., Lorusso, P., Granito, C., Ronzini, L., Troisi, L., Eur. J. Org. Chem. 2003, 68, 4053.

13. Florio, S., Lorusso, P., Granito, C., Luisi, R., Troisi, L. J. Org. Chem. 2004, 69, 4961.

14. (a) Wehrmeister, H. L.; J. Org. Chem. 1961, 26, 3821; (b) Hansen, J. F.; Wang, S. J. Org. Chem. 1976, 41, 3635.

15. Mąkosza, M., Staliński, K., Chemistry Eur. J. 1997, 3, 2025.

16. (a) Adam, W.; Mąkosza, M.; Staliński, K.; Zhao, C.G. J. Org. Chem. 1998, 63, 4390. (b) Surowiec, M.; Mąkosza, M. Tetrahedron 2004, 60, 5019.

17. Adam, W.; Bialas, J.; Hadjiarapoglou, L. Chem. Ber. 1991, 124, 2377. 